\title{
Investigation of the Downwash Induced by Rotary Wings in Ground Effect
}

\author{
Yasutada Tanabe* and Shigeru Saito* \\ Japan Aerospace Exploration Agency (JAXA) \\ Naoko Ooyama** and Katsumi Hiraoka*** \\ Department of Aeronautics and Astronautics, Tokai University \\ Kita-Kaname 1117, Hiratsuka, Kanagawa 259-1207, JAPAN Kanagawa, Japan
}

\begin{abstract}
There are concerns about the influence of the gust wind caused by helicopters affecting the moving vehicles while hovering over the road during rescue activities. For the understanding of such complicated flow, numerical simulation of a rotor hovering above the ground has been carried out, changing the rotor/ground clearances. The rotor thrust is kept constant, and the rotor control is determined by trim adjustments incorporated into the CFD algorithm. Collective pitch angle and the required power decreases with the rotor/ground clearance which agrees with experience. Changes of the flowfield near the rotor with regard to the rotor height are investigated based on the calculated results.
\end{abstract}

Keywords : Helicopter, Downwash, Hover, Ground effect, CFD, Moving overlapped grids

\section{Introduction}

Recently in Japan, the number of helicopters used for emergency medical services (EMS) and disaster relief has been increasing steadily year by year. It is proved to reduce the death-rate significantly, but there are still concerns about the influence of the downwash induced by the helicopter on nearby cars and motorcycles during landing and take-off on the highway.

More specifically, in Japan, EMS helicopter (called Doctor-Heli in Japan) were allowed to directly land onto the highway only very recently. The concern about the influence of the downwash on the ground traffic at the opposite lanes arises. A comparatively strict regulation of the ground traffic on the neighboring opposite lane is tentatively imposed. With clearer understanding of the structure of the helicopter downwash and its effect on the ground vehicles, hopefully a more reasonable regulation will be established which should improve the operation rate of the EMS helicopters in Japan further more. An investigation group was organized by ATEC (Association of Air Transport Engineering and Research) in Japan to study the detailed structure of the downwash caused by a hovering helicopter and its influence on the surface vehicles, especially motorcycles. Simulations using CFD methods were carried out in JAXA (Japan Aerospace eXploration Agency) for a hovering rotor above a flat plane at various heights from the ground [1] .

Away from the rotor, considering the similarity of the flowfield with the jet-blowing, it is proposed to switch to the jet-blowing experimental results [2] where the turbulent boundary layer developed on the ground is taken into consideration, while the swirl velocity

\footnotetext{
* Senior Researcher

** Graduate Student

*** Professor

E-mail :tan@chofu.jaxa.jp
}

Tel : $+82-422-40-3230$ 


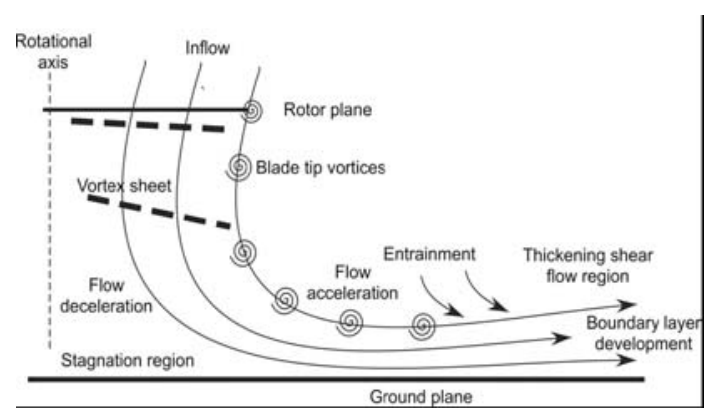

Fig. 1. General development of the flow below the rotor in ground effect operation [3]

component from the CFD results remains. It was suggested to use this simple downwash model of mean velocity for further analysis of the influence of the downwash from a hovering rotor on the ground traffic in the last paper by the present authors [1], but descriptions of flowfield in the near region of the rotor were absent. This paper is intended to focus on the changes of the flowfield near the rotor with regard to the rotor height based on the calculated results. Although the CFD method used here did not consider the viscous effects of the flow, it is considered that the flow in the near region of the rotor is not dominated by the viscous effect especially when the boundary layer on the ground is not developed.

When a helicopter is hovering in the air high enough from the ground, a high speed downwash is created by the rotor blades which can be generally predicted with simple momentum theory. This restraint of rotor downwash occurs as the helicopter reaches a relatively low altitude - usually one-half a rotor diameter. As the air is pushed downward by the main rotor system and semi-compressed against the surface, the net result is a beneficial increase in lift and a lower power requirement to support a given weight where the so-called IGE (in-ground effect) is observed. A simple schematic of the general flow developments is shown in Fig. 2 based on the observations by Lee et al [3]. On the ground plane, the flow becomes a wall jet with a boundary layer close to the surface and a developing shear flow region above with significant turbulent mixing. The flow structure changes significantly when the rotor height varies and high fluctuations of velocity along the ground are measured with PIV techniques. The experiment by Lee et al [3] is carried out with a very small rotor model $(\mathrm{R}=86 \mathrm{~mm})$ and the rotor tip speed was very low (Mtip $\sim 0.1$ ) compared with that of a conventional helicopter.

Considering the complexity, it is challenging to use a CFD method to correctly simulate and capture the main features of the flowfield induced by a hovering rotor in ground effect. The CFD method developed in JAXA is based on a moving overlapping grids approach and has been successfully applied to various flight conditions of an isolated rotor or with other components of a helicopter [4]. It is the first time this approach is used to simulate the flow induced by a rotor in ground effect. The required power reduction from IGE is well predicted so as the mean velocities while a low frequency unsteadiness in the flowfield is observed. Several lessons were also learned for further improvement of the prediction of this complicated flow structure especially near the ground.

\section{Numerical Method}

A CFD code referred as JAXA_ov3d [3] based on the moving overlapped grids approach is used and its main features are as follows:

- A fine inner background Cartesian grid is used to resolve the vortex wake of the rotor in addition to the wider outer background Cartesian grid. SOH type blade grid is used mainly for shape fidelity. 
- $\quad$ Divided time steps between grids allow correct time advancing based on blade azimuth angle under respective CFL number limits set for each grid where different numerical schemes can be used.

- $\quad$ Fully unsteady Euler/NS formulations are applied so that the blade motion effects are reflected through grid moving and deforming only.

- A Simple High-resolution Upwind Scheme (SHUS) [5] is used for the Cartesian background grids with $4^{\text {th }}$ order accuracy in space and also an explicit 4 stages Runge-Kutta integration scheme [6] in time is used.

- A robust 2nd-order implicit TVD scheme [7] is used for the moving blade grids allowing larger CFL numbers.

- $\quad$ Standard MPI routines are adapted for parallel computing processes.

As shown in Fig. 2, a moving overlapped grid system is used for this study. The ground boundary condition is applied to the bottom surface of the outer background grid. The height of the rotor from the ground is simulated by changing the position of the bottom surface relative to the center of the rotor. The HART II rotor model [8] which simulates the main rotor of $\mathrm{BO}-105$ helicopter is used conveniently for this study. The rotor height $h_{R}$ is changed from $0.6 \mathrm{R}$ to $3 \mathrm{R}$ and also an out-of-ground effect case is calculated for reference as shown in Table 1.

In this study, the blade grid points are 51 in the radial direction, 95 in the chordwise direction and 25 in the thickness direction. Four blade grids are used. The inner background grid is automatically constructed as a uniform distributed Cartesian mesh at each direction. The range of the inner background grid is $-1.4 R \sim+1.4 R$ in the $\mathrm{X}$ and $\mathrm{Y}$ directions discreted with 151 points and $-0.4 R \sim+0.4 R$ in the $Z$ direction discreted with 51 points.

Table 1. IGE computation cases

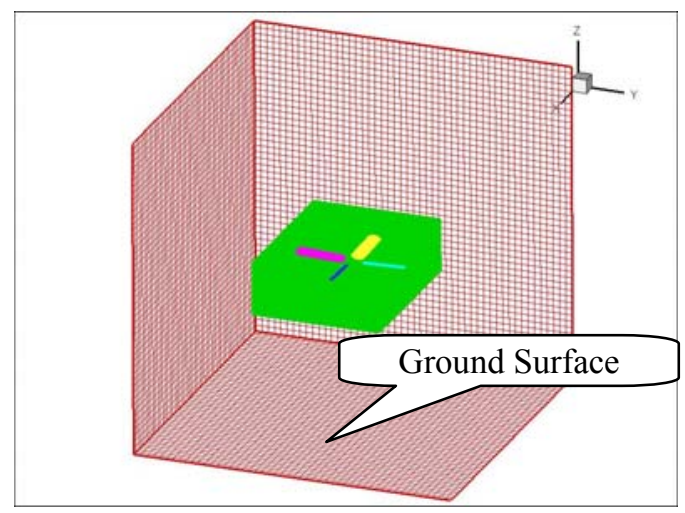

\begin{tabular}{|c|c|c|}
\hline Case No. & $h_{R} / R$ & CFD Domain \\
\hline 1 & 0.6 & \multirow{9}{*}{$\begin{array}{c}-6.0 \leqq X / R \leqq 6.0 \\
-6.0 \leqq Y / R \leqq 6.0 \\
-h_{R} / R \leqq Z / R \leqq 3.0\end{array}$} \\
\hline 2 & 0.8 & \\
\hline 3 & 0.9 & \\
\hline 4 & 1.0 & \\
\hline 5 & 1.2 & \\
\hline 6 & 1.5 & \\
\hline 7 & 2.0 & \\
\hline 8 & 3.0 & \\
\hline 9 & OGE & \\
\hline
\end{tabular}

Fig. 2. Overlapped grid system used for ground effect simulation

Table 2. Computation conditions of HART II rotor model in hover

\begin{tabular}{|l|l|}
\hline \multicolumn{2}{|c|}{ HART II Model } \\
\hline Rotor Radius & $R=2 \mathrm{~m}$ \\
\hline Blade chord & $c=0.121 \mathrm{~m}$ \\
\hline Blade number & $\mathrm{N}_{\mathrm{BLD}}=4$ \\
\hline Freestream Mach number & $\mathrm{M}_{\infty}=0.0$ \\
\hline Hovering tip Mach number & $\mathrm{M}_{\mathrm{tip}}=0.6347$ \\
\hline Rotation speed & $\Omega=109.12 \mathrm{rad} / \mathrm{s}$ \\
\hline Advance ratio & $\mu=0.0$ \\
\hline Rotor height from ground & $h_{R} / R=0.6 \sim 3.0$ \\
\hline & $T=3300 \mathrm{~N}$ \\
Thrust & $T_{T}=0.004574$ \\
\hline & $C_{T} / \sigma=0.004574$ \\
\hline
\end{tabular}


The corresponding resolutions in each directions are $\Delta X_{B}=\Delta Y_{B}=0.0187 R$ and $\Delta Z_{B}=0.016 R$. The outer background grid is also automatically constructed as a uniform distributed Cartesian mesh at each direction. The range of the outer background grid is $-6 \mathrm{R} \sim+6 \mathrm{R}$ in the $\mathrm{X}$ and $\mathrm{Y}$ directions discreted with 201 points and $-h_{R} \sim+3 R$ in the $\mathrm{Z}$ direction discreted with 101 points. The corresponding resolutions in each directions are $\Delta X_{O B}=\Delta Y_{O B}=0.06 R$ and $\Delta Z_{O B}=0.036 R \sim 0.06 R$ for $h_{R}=0.6 R \sim 3 R$.

During the computation, the collective pitch angle of the rotor is adjusted every 90 degrees of azimuth angle to keep the averaged thrust per revolution equal to the target thrust. The rotor blades abruptly start to rotate at a constant speed at the beginning of the computation. The ground effect is felt by the blade after several rotor revolutions depending on the rotor height where a smaller pitch angle is enough to produce a same thrust in this case. The collective pitch angles and required torques corresponding to different rotor heights are obtained under a constant thrust condition as shown in Table 2.

$C_{T}$ is defined in this numerical simulation as

$$
C_{T}=T / \rho A(R \Omega)^{2}
$$

\section{Results and Discussion}

The calculated values of the collective pitch angle with regard to various rotor heights to maintain a constant thrust are shown in Fig. 3 Because of the unsteadiness of this flowfield, the collective pitch angle is shown with its variation range as an error bar to its mean value in the figure.

The torque coefficient is defined as

$$
C_{Q}=Q / \rho A(R \Omega)^{2} R
$$

Fig. 4 shows the calculated required power reduction from the OGE condition versus the rotor height. The result compares well with Cheeseman-Bennett' s relation [9].

$$
\left(\frac{P_{I G E}}{P_{O G E}}\right)_{T=\text { const }}=\frac{1}{1+\left(R / 4 h_{R}\right)^{2}}
$$

Hayden' empirical relation [10] defined as

$$
\left(\frac{P_{I G E}}{P_{O G E}}\right)_{T=\text { const }}=\frac{1}{0.9926+0.03794\left(2 R / h_{R}\right)^{2}}
$$

is also shown in Fig. 4. This empirical relation is based on the helicopter flight test data where the fuselage exists. As can be seen from Fig. 6-9, where the velocity vectors in the rotor center section are shown, there is a significant upwash area in the center part of the rotor which becomes stronger when the rotor height decreases, that should benefit the power consumption when a fuselage is located there.

Velocity vectors in the $\mathrm{X}$ plane for a rotor height of 1.0R are shown in Fig. 5. The velocity vectors vary with the blade azimuth position. The velocity vectors shown in Figure $5-11$ are when the blade is in the same plane after 30 revolutions from the calculation start and the influence of the starting vortex can be excluded. Close views of velocity vectors at the rotor center section for six rotor heights off ground, $h_{R} / R=0.6,0.9,1.0$ and 1.2 are shown in Fig. 6-11. It can be seen the high speed flow is constrained in a thin layer along the ground surface which forms a wall jet. With lower rotor height, larger upward velocity across the rotor blade can be observed. 


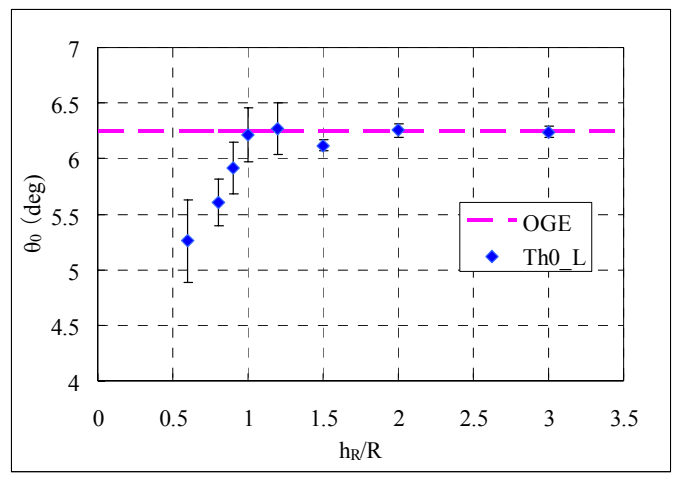

Fig. 3. Required collective pitch angle vs rotor height, error bars indicate the range of max and min values

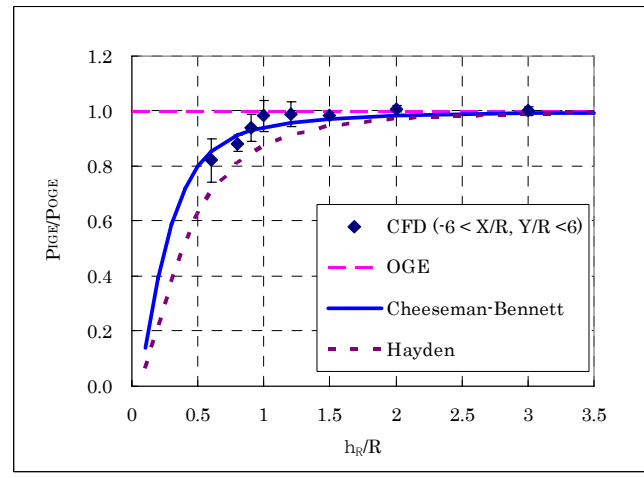

Fig. 4. Required power change with rotor height, error bars indicate the range of max and min values

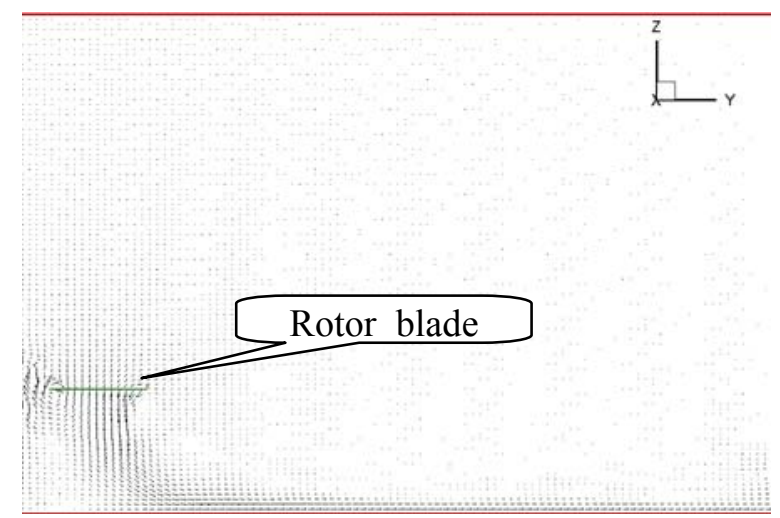

Fig. 5. Velocity vectors in the X plane for $h_{R} / R=1.0$

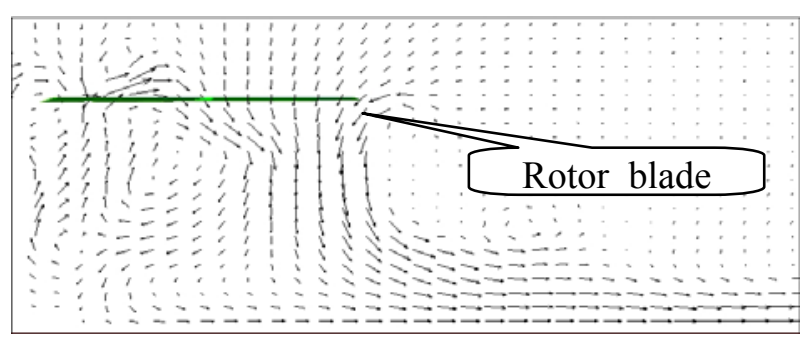

Fig. 6. Close view of Velocity vectors in the $\mathrm{X}$ plane for $h_{R} / R=0.6$

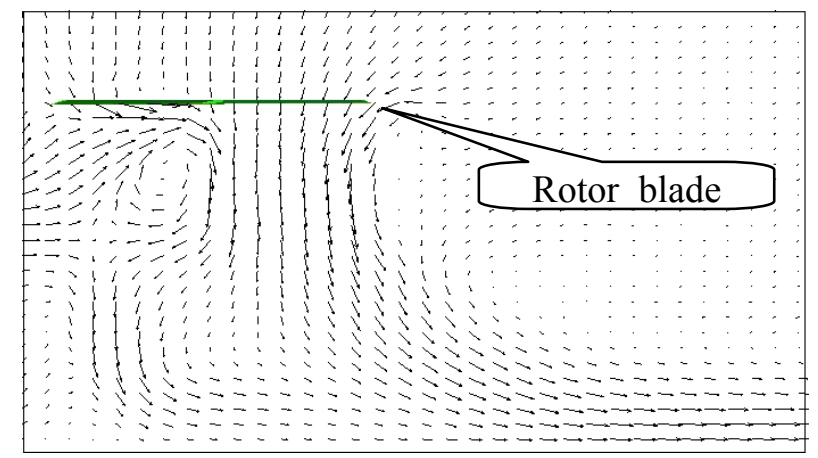

Fig. 7. Close view of Velocity vectors in the X plane for $h_{R} / R=0.9$ 


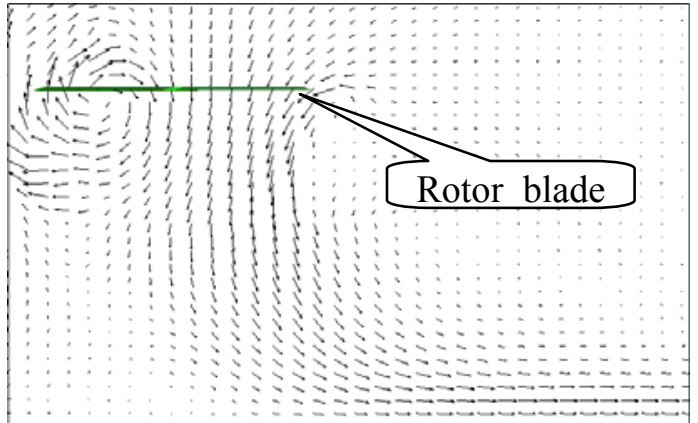

Fig. 8. Close view of Velocity vectors in the $\mathrm{X}$ plane for $h_{R} / R=1.0$

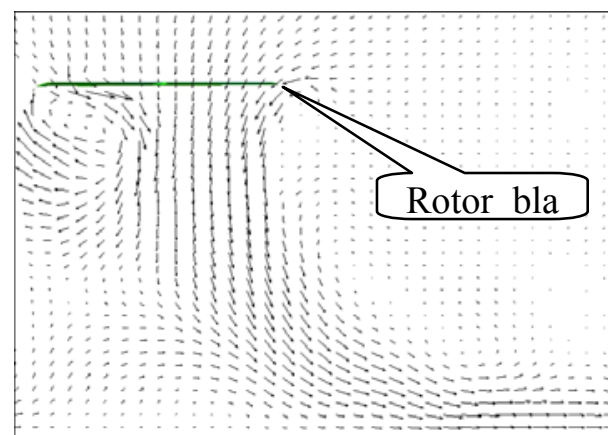

Fig. 9. Close view of Velocity vectors in X plane for $h_{R} / R=1.2$

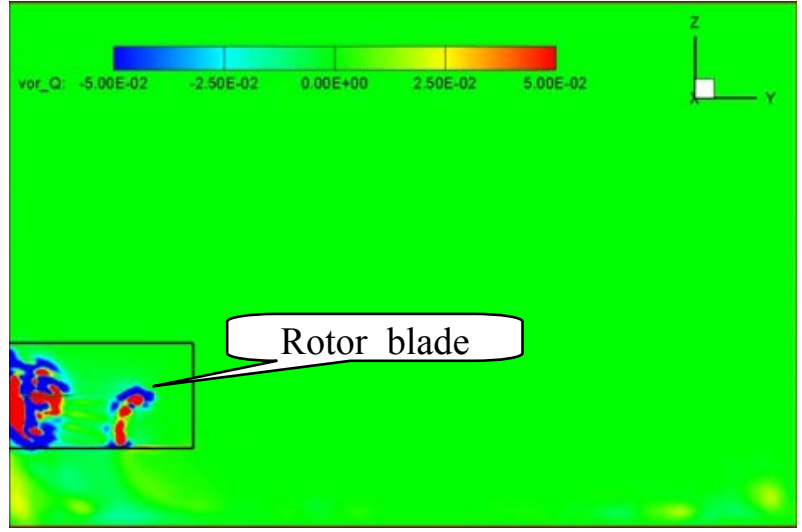

Fig. 10. Q-criterion contour in the $\mathrm{X}$ plane for $h_{R} / R=1.0$

The $\mathrm{Q}-$ criterion contour for $h_{R} / R=1.0$ is plotted in the $\mathrm{X}$ plane as shown in Fig. 10. The $\mathrm{Q}$-criterion value corresponds to the rotational component of the vortex and is used to visualize the blade tip vortex with better resolution. The rectangular frame indicates the boundary of the inner background grid. As can be seen from the figure, blade tip vortices are well captured inside the inner background grid, but diffuse abruptly into the outer background grid. The method applied to interpolate between the grids is the trilinear interpolation and the conservation between these overlapped grids can not be automatically satisfied. Nearly equal grid densities are desirable to obtain high quality interpolations. In the present calculations, the grid size in outer background grid is about 3.2 times that of the inner background grid. Smoother grid size transition or a much denser grid in the outer background grid should be used to preserve the tip vortex and simulate the vortex / ground interference better. This is one of the lessons learned from the current study. However, as shown in Fig. 15, the radial velocity profiles for the rotor height $h_{R} / R=1.0$ agrees well with the experimental data by Iboshi et al.[11]. It is considered that the mean velocities are transferred into the outer background grid with good accuracy and the obtained mean flowfields of the rotor in ground effect are valid.

Close views of the $\mathrm{Q}$-criterion contour in the $\mathrm{X}$ plane for $h_{R} / R=0.6 \sim 1.2$ are shown in Fig. $11 \sim 14$. It can be seen from these figures that the distance between tip vortices at lower rotor height is more compressed and the $3^{\text {rd }}$ and $4^{\text {th }}$ tip vortex tends to merge at these rotor heights.

Radial velocity profiles for rotor height $h_{R} / R=1.0$ with comparison with existing experimental data by Iboshi et al.[11] are shown in Fig. 15. The velocity is nondimensionalized by the uniform induced velocity defined as 


$$
V_{i 0}=\sqrt{\frac{T}{2 \rho A}}=\sqrt{\frac{m g}{2 \rho \pi R^{2}}}
$$

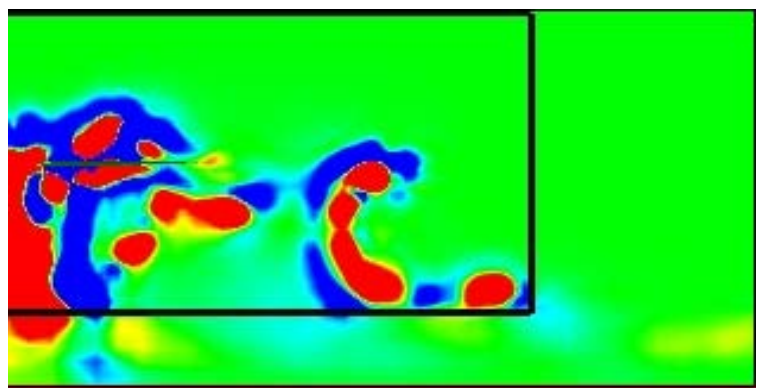

Fig. 11. Close view of $\mathrm{Q}$-criterion contour in the $\mathrm{X}$ plane for $h_{R} / R=0.6$

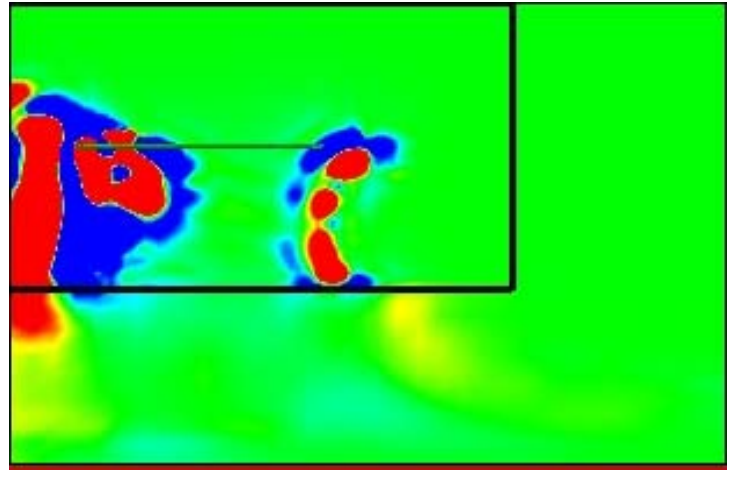

Fig. 12. Close view of $Q-$ criterion contour in the X plane for $h_{R} / R=0.9$

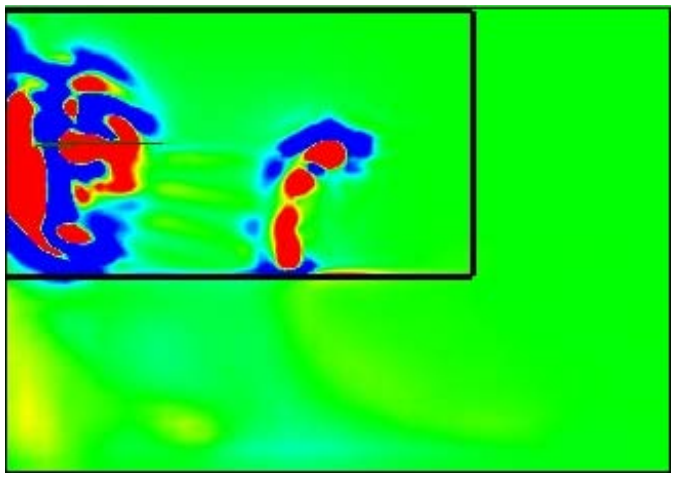

Fig. 13. Close view of $Q-$ criterion contour in the $\mathrm{X}$ plane for $h_{R} / R=1.0$

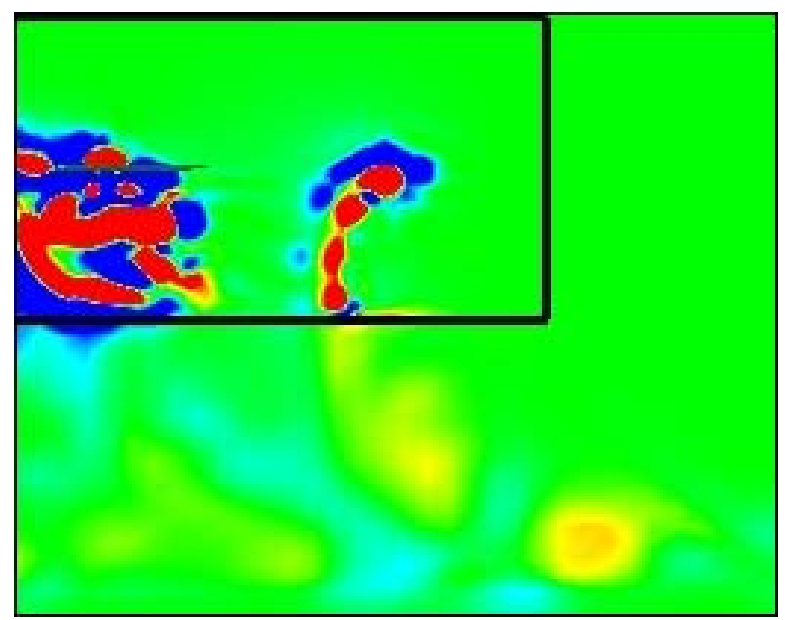

Fig. 14. Close view of $\mathrm{Q}$-criterion contour in the $\mathrm{X}$ plane for $h_{R} / R=1.2$

Apparently, due to the neglect of the viscous effects in the CFD Euler solution, boundary layer development on the ground surface for the wall jet can not be predicted. The velocity profile at $\mathrm{X} / \mathrm{R}=3.0$ is poor for that reason. However, at the near positions from the rotor, the comparison of the CFD results with the experiment is quite good. 


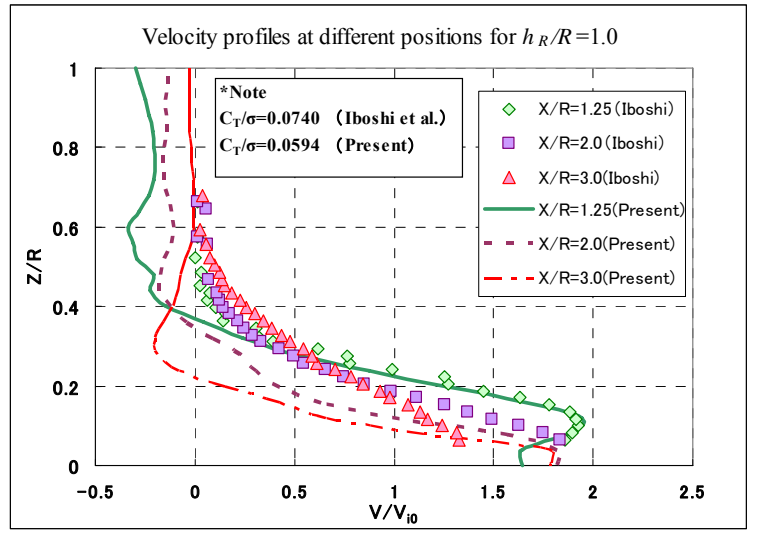

Fig. 15. Comparison of velocity profiles between computation and experiment

Phase-Averaged velocity profiles calculated using the CFD Euler solver for six rotor heights off ground at $\mathrm{Y} / \mathrm{R}=0.6,1.2$ and 1.8 are shown in Fig. 16 18. The radial velocity profiles at $\mathrm{Y} / \mathrm{R}=0.6$ in Fig 16 have not developed in the radial direction yet, due to the wake flow just after blowing down from the rotor. It seems that the flow speed just under the rotor is not high but has complicated recursive flow patterns. At $\mathrm{Y} / \mathrm{R}=1.8$, as shown in Fig. 18, wall jets are formed where a high speed flow (about twice the induced velocity) is concentrated in a thin layer on the ground. The data shown in Fig. 18 is obtained when the rotor is at 30 revolutions from the start.

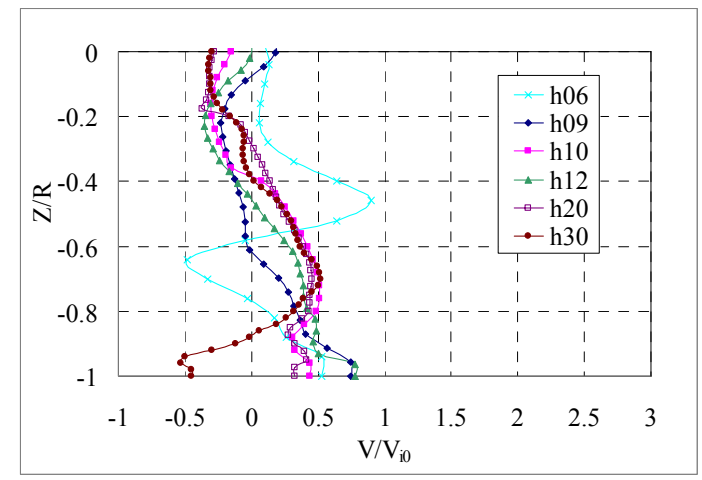

Fig. 16. Phase-Averaged velocity profiles at $Y / R=0.6$

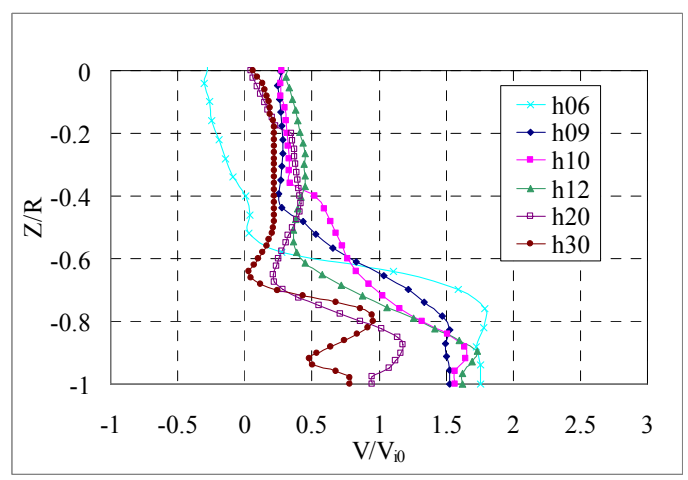

Fig. 17. Phase-Averaged velocity profiles at $Y / R=1.2$

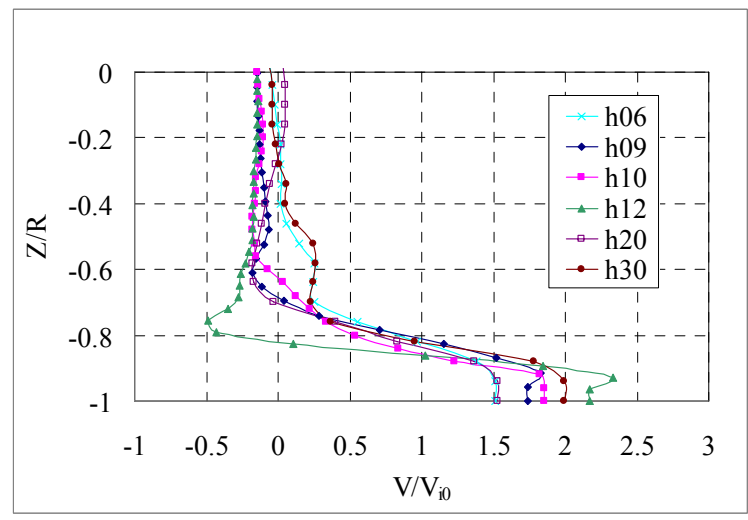

Fig. 18. Phase-Averaged velocity profiles at $Y / R=1.8$ 


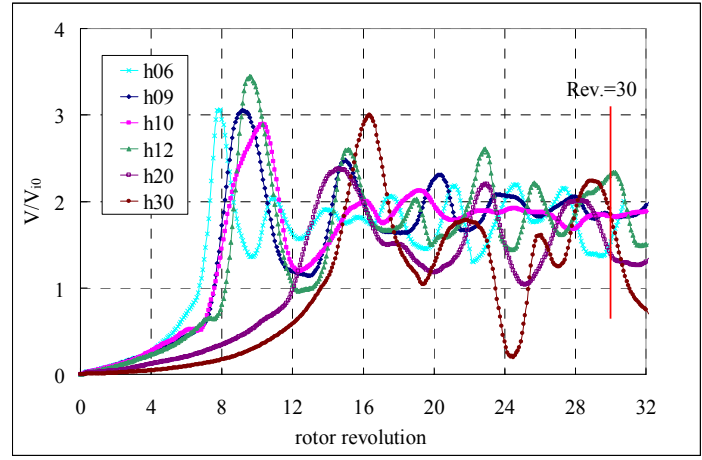

Fig. 19. Wall speed fluctuations at $Y / R=1.8$

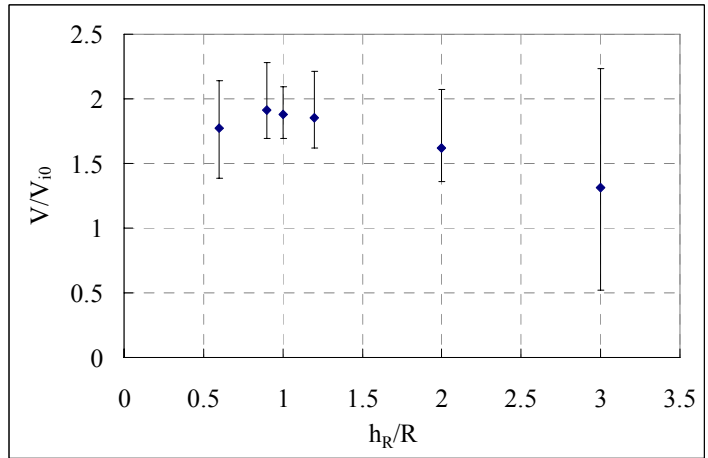

Fig. 20. Mean wall speed at $\mathrm{Y} / \mathrm{R}=1.8$, error bars indicate the range of max and min values

As a check of the periodicity of the flowfield, the time histories of wall speeds at $\mathrm{Y} / \mathrm{R}=1.8$ are shown in Fig. 19. The wall speeds fluctuate with large amplitudes and the periodicities are poor. The reason of the failure to obtain generally expected periodic solutions may come from the rotor trim adjustment method used in the present study which needs to be studied further. Same phenomenon is observed in Ref. [12] and it is considered revealing the characteristic low-frequency unsteadiness of the rotor flowfields in ground effect. The mean speeds with respect to rotor height are shown in Fig. 20 together with their variation range. It is interesting that the maximum mean velocity occurs at $h_{R} / R=0.9$ rather than 0.6. In reference 3 , the same trend was found by Lee et al. experimentally where $h_{R} / R=1.0$ was the highest.

As a measurement of the flowfield unsteadiness time scale, the fluctuation periods around the mean value from Fig. 19 are plotted in Fig. 21 for each rotor height. Although it seems that a lower rotor height gives a shorter period of fluctuation, the correlation is not good. Especially for $h_{R} / R=3.0$, where the ground effect is slight as felt by the rotor, the fluctuation period on the ground becomes shorter again, indicating that different physics that cause these fluctuations may exist. It must be mentioned that because the collective pitch angle is adjusted 4 times per revolution to maintain a constant thrust, this computation is similar to a hovering flight simulation. The influence of the adjustment period of the collective pitch angle on the flowfield fluctuation period is unknown at this point and needs further studies. The reaction delays defined as the time from computation start where the wall speed reaches a value of the induced velocity are also plotted in Fig.21, which shows a good linearity with rotor height.

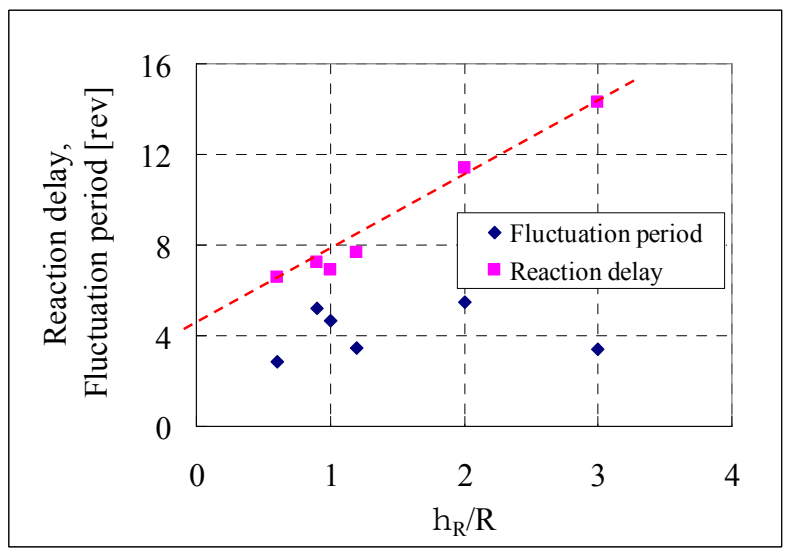

Fig. 21. Fluctuation period at $Y / R=1.8$ 


\section{Concluding Remarks}

Downwash caused by hovering rotors in ground effect is studied with focus on its flow structure in the near region of the rotor.

With a Euler solver, a rotor in ground effect is simulated and the obtained power merit curve for an isolated rotor agreed with the Cheeseman-Bennett relation.

The rotor height has significant influence on the flowfield structure in the near range of the rotor.

Strong unsteadiness in the flowfield is observed. The mean wall speed in the near range of the rotor has a maximum at an intermediate rotor height which agrees with other experimental observations.

The fuselage can have strong influence on the near-field flow structure and was not taken into account for the current study. Further CFD studies including viscous effects and fuselage could give more accurate and detailed flowfield predictions. Finer grid resolution in the flowfield where the tip vortices exist and near the ground would be required to predict the unsteadiness in the flowfiled with better accuracy.

\section{Acknowledgments}

The authors would like to acknowledge all the members who participated the ATEC working group for "Research of the Influence of Helicopter Downwash on Ground Traffics". Members are from the major helicopter operators and research institutes in Japan. Professor Iboshi of Japan National Defense Academy provided his experimental data for comparisons. Without their inspiration and advices, this study would not be initiated.

\section{References}

1. Tanabe, Y., Saito, S., Ooyama, N. and Hiraoka, K., Study of a Downwash Caused by a Hovering Rotor in Ground Effect, $34^{\text {th }}$ European Rotorcraft Forum, Liverpool, UK, September 16-18, 2008.

2. Rajaratnam N., Turbulent Jets, Elsevier Scientific Publishing Company, Amsterdam, 1976.

3. Lee, T.E., Leishman, J.G. and Ramasamy, M., Fluid dynamics of interacting blade tip vortices with a ground plane, $64^{\text {th }}$ Annual Forum of AHS, Montreal, Canada, April 29-May 1, 2008.

4. Tanabe, Y. and Saito, S., An integrated analysis code with CFD/rotor dynamics coupling developed in JAXA, AHS Specialists' Conference on Aeromechanics, San Francisco, CA, January 23-25, 2008.

5. Shima, E., and Jounouchi, T., Role of CFD in aeronautical engineering (No.14) AUSM type upwind schemes - , NAL SP-34, 1999, pp.7-12.

6. Jameson, A., Schmidt, W., and Turkel, E., Numerical solutions of the Euler equations by finite volume method using Runge-Kutta time stepping schemes, AIAA Paper 81-1259, 1981.

7. Yee, H.C., and Harten, A., Implicit TVD schemes for hyperbolic conservation laws in curvilinear coordinates, AIAA Journal, Vol. 25, No.2, February 1987, pp.266-274.

8. Y.H. Yu, C. Tung, B. van der Wall, H.-J. Pausder, C. Burley, T. Brooks, P. Beaumier, Y. Delrieux, E. Mercker, and K. Pengel, The HART-II test: rotor wakes and aeroacoustics with Higher-Harmonic pitch Control (HHC) inputs - the joint German/French/Dutch/US project -, AHS 58th Annual Forum, Montreal, Canada, June 11-13, 2002.

9. Cheeseman I.C. and Bennett W.E., The effect of the ground on a helicopter rotor, R\&M 3021, ARC, 1955.

10. Hayden J.S., The effect of the ground on helicopter hover power required, AHS $32^{\text {nd }}$ Annual Forum, Washington, DC, May 1976.

11. Iboshi N., Usuda H. and Itoga N., Ground effect of a hovering rotor over confined area, $45^{\text {th }}$ Aircraft Symposium, Kitakyushu, Japan, October 10-12, 2007. (In Japanese).

12. Phillips C. and Brwon R., Eulerian Simulation of the Fluid Dynamics of Helicopter Brownout, AHS 64 ${ }^{\text {th }}$ Annual Forum, Montreal, Canada, April 29-May1, 2008. 